\title{
Zinc Indiffused Diced Ridge PPLN Waveguides for Photorefractive Damage Resistance and Spectral Engineering
}

\author{
Sam A. Berry ${ }^{1}$, Lewis G. Carpenter ${ }^{1}$, J. C. Gates ${ }^{1}$, Peter, G. R. Smith ${ }^{1,2}$, Corin B. E. Gawith ${ }^{1,2}$ \\ 1. Optoelectronics Research Centre, University of Southampton, Southampton SO17 1BJ, United Kingdom \\ 2. Covesion Ltd., Premier Way, Romsey SO51 9DG, United Kingdom
}

Nonlinear devices have been essential for extending laser wavelength operating ranges since its invention. The ability to convert a laser's wavelength between the telecoms C-band and $780 \mathrm{~nm}$ has attracted much attention recently due to the developments of magneto-optical traps using $\mathrm{Rb}$ aimed at high precision gravity measurements and quantum optics for generating entangled photon pairs. For magneto-optical traps the low linewidths and low cost optical components from well established telecoms technology have enabled the efficient generation of all the small frequency shifts required to address the excitation states of the Rb. Nonlinear waveguides based on direct bonded lithium niobate on lithium tantalate have demonstrated highest efficiency second harmonic generation (SHG) of telecoms lasers [1], but are difficult to fabricate. Ti indiffused waveguides have been shown as an alternative, but it has been shown recently that photorefraction limits the performance of these waveguides to less than $10 \mathrm{~mW}[2]$.

Our $\mathrm{Zn}$ indiffused waveguides are realised by creating a waveguide directly from periodically poled lithium niobate and have $<2 \mathrm{~dB}$ insertion loss. The waveguide's vertical confinement is created by a zinc based indiffusion at a temperature below the crystal's Curie point and the lateral confinement is created by physical machining in the ductile regime to create optical quality cuts [3]. In contrast to Ti indiffusion, the presence of $\mathrm{Zn}$ in the nonlinear crystal reduces its susceptibility to photorefractive damage [1,2], enabling output powers $>100 \mathrm{~mW}$.

Fig 1(a) shows a typical SHG spectra measured when launched with $300 \mathrm{~mW}$ of input power, compared with a measured spectra (right) from a custom apodised grating waveguide which suppresses the sinc lobe features. Fig 1(b) shows SHG output vs. launched $1560 \mathrm{~nm}$ light for one of our $40 \mathrm{~mm}$ long waveguides using a commercial EDFA seeded with an tunable laser; the inset shows the output spectra at the maximum power of $925 \mathrm{~mW}$ launched with a peak SHG output of $130 \mathrm{~mW}$. Fig 1(c) show a photograph of one of our $40 \mathrm{~mm}$ devices, the illuminating light is highlighting the presence of the periodically poled domain structure towards the right.

We will present our latest work on $\mathrm{Zn}$ indiffused ridge waveguides with approaches for increasing the efficiency at higher powers and customising the spectral output using apodization of the periodically poled domain structure.
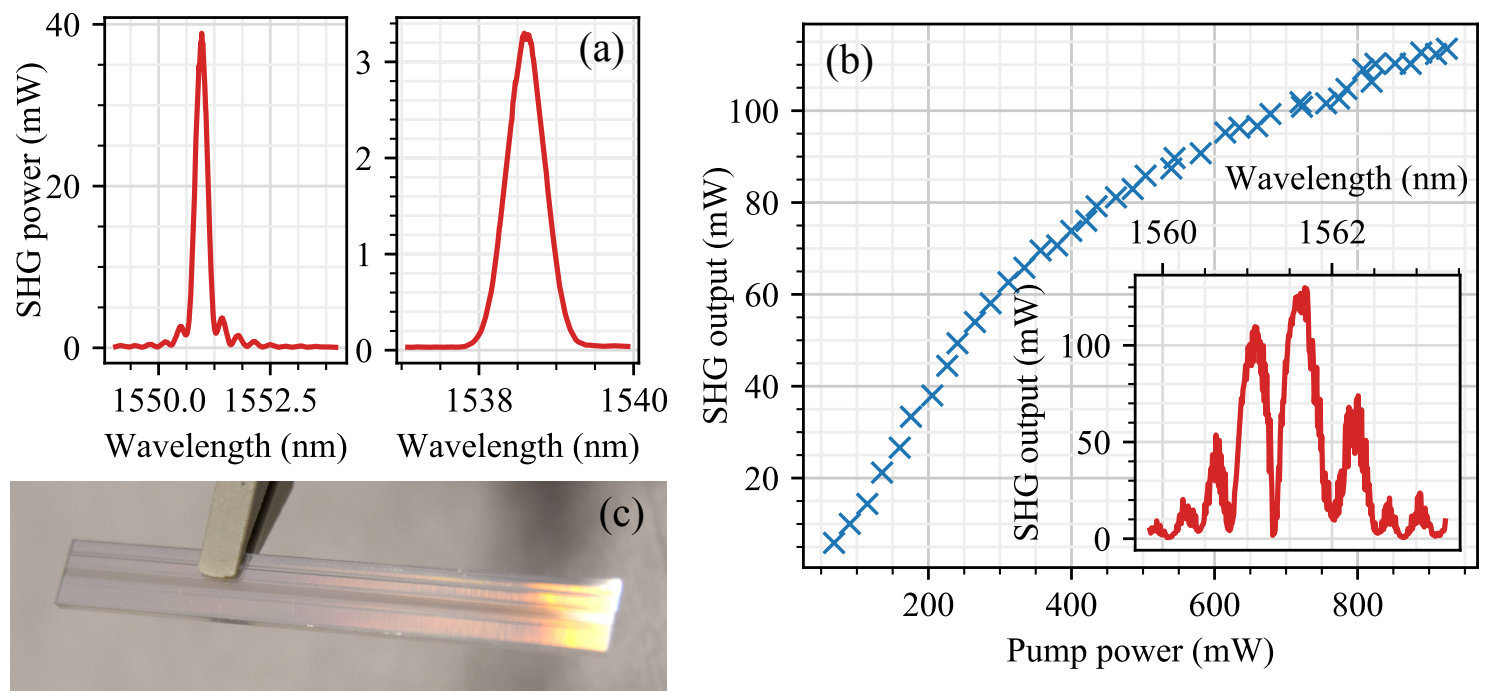

Fig. 1 (a) SHG output spectra from the PPLN waveguide with $300 \mathrm{~mW}$ of input power. (b) The SHG output scaling with launched pump power (inset shows the spectra at $925 \mathrm{~mW}$ pump power). (c) A photograph of one of the $40 \mathrm{~mm}$ chips containing $5 \mathrm{Zn}$ indiffused ridge waveguides.

\section{References}

[1] T. Umeki, O. Tadanaga, and M. Asobe, "Highly Efficient Wavelength Converter Using Direct-Bonded PPZnLN Ridge Waveguide," IEEE J. Quantum Electron., 46, 1206 (2010).

[2] S. Pal, B. Das, and W. Sohler, "Photorefractive damage resistance in Ti:PPLN waveguides with ridge geometry," Appl. Phys. B, 120, 737 (2015)

[3] L.G. Carpenter, S. A. Berry, and C.B.E. Gawith, "Ductile dicing of LiNbO3 ridge waveguide facets to achieve $0.29 \mathrm{~nm}$ surface roughness in single process step," Electron. Lett., 53, 1672-1674 (2017). 